\title{
Metabolic Efficiency of Sugar Co-Metabolism and Phenol Degradation in Alicyclobacillus acidocaldarius for Improved Lignocellulose Processing
}

\author{
Ashley E. Beck ${ }^{\mathbb{D}}$ \\ Biological and Agricultural Engineering, North Carolina State University, Raleigh, NC 27695, USA; \\ aebeck@ncsu.edu; Tel.: +1-919-515-6746
}

Received: 31 March 2020; Accepted: 20 April 2020; Published: 27 April 2020

\begin{abstract}
Substrate availability plays a key role in dictating metabolic strategies. Most microorganisms consume carbon/energy sources in a sequential, preferential order. The presented study investigates metabolic strategies of Alicyclobacillus acidocaldarius, a thermoacidophilic bacterium that has been shown to co-utilize glucose and xylose, as well as degrade phenolic compounds. An existing metabolic model was expanded to include phenol degradation and was analyzed with both metabolic pathway and constraint-based analysis methods. Elementary flux mode analysis was used in conjunction with resource allocation theory to investigate ecologically optimal metabolic pathways for different carbon substrate combinations. Additionally, a dynamic version of flux balance analysis was used to generate time-resolved simulations of growth on phenol and xylose. Results showed that availability of xylose along with glucose did not predict enhanced growth efficiency beyond that of glucose alone, but did predict some differences in pathway utilization and byproduct profiles. In contrast, addition of phenol as a co-substrate with xylose predicted lower growth efficiency. Dynamic simulations predicted co-consumption of xylose and phenol in a similar pattern as previously reported experiments. Altogether, this work serves as a case study for combining both elementary flux mode and flux balance analyses to probe unique metabolic features, and also demonstrates the versatility of $A$. acidocaldarius for lignocellulosic biomass processing applications.
\end{abstract}

Keywords: co-metabolism; dynamic flux balance analysis; elementary flux mode analysis; resource allocation; thermoacidophile

\section{Introduction}

Alicyclobacillus acidocaldarius is a thermoacidophilic bacterium originally isolated from Yellowstone National Park [1] of interest for its thermostable enzymes [2,3] as well as its unique physiology and ability to thrive in low $\mathrm{pH}$ environments [4,5]. It has also been linked to fruit or juice spoilage due to acid production [6]. This microorganism was originally classified as Bacillus acidocaldarius and later transferred to the newly created genus Alicyclobacillus due to the unique $\omega$-alicyclic fatty acids and hopanoids composing the phospholipid membrane and 16S rRNA classification [7]. Recent studies have discovered additional unique capacities of $A$. acidocaldarius, including the ability to simultaneously metabolize hexose and pentose sugars [8], as well as the ability to degrade toxic phenolic compounds [9]. These findings provide a unique potential role for this microorganism in lignocellulosic biomass bioprocessing, as biomass feedstocks typically contain both hexose and pentose sugar units, as well as phenolic lignin derivatives, which are toxic to many microbial catalysts used in processing [10]. An organism that is able to break down toxic compounds and concurrently utilize multiple sugar types will be of great aid in biomass processing, with applications in bioenergy and biochemical production, as well as remediation of other industrial wastes. 
A Gram-positive, spore-forming obligate aerobe, A. acidocaldarius has been shown to grow on a variety of carbon sources, including glucose, fructose, mannose, ribose, arabinose, xylose, galactose, rhamnose, glycerol, mannitol, methylglucoside, arbutin, aesculin, salicin, cellobiose, maltose, lactose, melibiose, sucrose, trehalose, raffinose, starch, and glycogen [11]. This organism has the ability to degrade hemicellulose and cellulose [12,13], making it very useful for biomass processing applications. In particular, energy-limited chemostat growth on glucose has been characterized under different temperatures and $\mathrm{pH}[14,15]$. A recent continuous culture study has further characterized the ability of this microorganism to simultaneously metabolize two different sugars under non-carbon-limiting conditions-glucose and xylose, arabinose and xylose, and fructose and glucose [8], indicating that $A$. acidocaldarius does not operate according to conventional carbon catabolite regulation, in which carbon sources are typically consumed sequentially in order of preference [16,17].

Pretreatment of lignocellulosic biomass liberates sugars (e.g., disaccharides and monosaccharides) from recalcitrant polymers for easier metabolism by microbial catalysts [18]. However, lignin is a common component of most biomass feedstocks (ranging from 6-29 percent varying by feedstock type [19]) and is not suitable for most microbial catalysts used in biomass processing, with pretreatment steps resulting in inhibitory or toxic lignin derivatives, including furans, organic acids, and phenolic compounds [19-21]. Another recent study has investigated the tolerance of A. acidocaldarius to selected phenolic compounds derivatized from lignin, including phenol and ferulic, $\rho$-coumaric, and sinapinic acids, common aromatic compounds [9]. Phenolic compounds are also produced as byproducts from many other agricultural and industrial chemical processes and often end up as downstream pollutants in water bodies [22-24]. This previous study demonstrated the ability of A. acidocaldarius to tolerate and degrade each of the four tested phenolic compounds in the presence of xylose as the primary energy source. Degradation of all four compounds was enhanced by the presence of copper, indicating the likely role of putative multicopper oxidases identified in the genome [9].

The ability of $A$. acidocaldarius to simultaneously metabolize pentose and hexose sugars as well as degrade toxic phenolic compounds demonstrates a versatile metabolic potential that can be harnessed for improved biomass processing efficacy and economic feasibility. With the onset of genomics and data-intensive science, metabolic modeling is becoming more commonly used and has proven to be a powerful tool to probe complex systems, understand metabolic pathways in silico, and provide directives for experimental work, as well as potential metabolic engineering strategies [25-28]. Stoichiometric metabolic modeling methods use genomic information to compile the set of metabolic reactions that an organism has the capacity to perform, curated by literature and biochemical knowledge, to quantitatively describe and analyze an organism's metabolism ab initio based on genetic potential [29]. This set of reactions is depicted mathematically in the form of a stoichiometric matrix, which represents a mass-balanced metabolism. Constraint-based and metabolic pathway analysis techniques can then be applied to solve the stoichiometric matrix for steady-state reaction fluxes. Flux balance analysis (FBA) is one of the most commonly applied methods and employs a linear programming optimization algorithm to determine a flux solution to the stoichiometric matrix that optimizes a predetermined cellular objective constraint, e.g., maximization of biomass [30,31]. Elementary flux mode analysis (EFMA) is a metabolic pathway analysis method that enumerates the collection of all possible core routes (elementary flux modes, EFMs) through a metabolic network [32,33]. These core routes are minimal sets of enzymatic reactions that transform substrates to products, and this collection of EFMs characterizes the entire phenotypic space. Rather than providing a single optimal flux solution based on a cellular objective criterion, EFMA allows an unbiased examination of metabolic capabilities [34]. Resource allocation theory can then be incorporated to interpret predicted pathways (e.g., efficiency with respect to different nutrients) and thus comprehensively investigate metabolic acclimations under varying degrees of stress. Such systematic characterization of the phenotypic space provides an ecological basis for understanding metabolic pathways [35-37].

While much of the previous work on A. acidocaldarius has centered around its cellulose degradation capabilities (e.g., using thermostable endoglucanases for enzymatic pretreatment of biomass [12]), 
the recent studies described above have opened up new research avenues to expand the contributions of this microorganism to the lignocellulose processing industry through co-metabolism of hexose and pentose sugars [8] and the ability to tolerate and degrade inhibitory phenolic compounds [9]. A. acidocaldarius is known to produce organic acids and has been reported to produce lactate, acetate, and propionate [8], which can be incorporated in biochemical production platforms. Another recent study identified $A$. acidocaldarius as a dominant bacterial member colonizing a sugarcane bagasse stockpile [38], indicating that it may have important implications in microbial community interactions, as well as hold potential for industrial processing applications. In the current study, both EFMA and a dynamic version of FBA were used to understand and quantify the potential benefits and ramifications of substrate co-metabolism in A. acidocaldarius, specifically to analyze metabolic pathways in terms of energetic efficiency and operating/investment costs used for sugar catabolic pathways with the aim to better understand the impact of a lack of carbon catabolite regulation, as well as degradation of phenolic compounds. The roles of oxygen and overall pathway investment were investigated in relation to carbon consumption efficiency for different substrate combinations. In addition, the existing metabolic model for A. acidocaldarius was expanded to include the phenol degradation pathway and analyze the metabolic efficiencies of phenol degradation co-occurring with sugar consumption. Based on previously measured kinetic parameters for growth on xylose with phenol [9], the metabolic model was analyzed with dynamic FBA to examine the optimum carbon consumption pathways in this organism.

\section{Materials and Methods}

\subsection{Metabolic Model}

A metabolic model for A. acidocaldarius was previously constructed and published by Beck et al. [39] with experimentally measured biomass composition demonstrating analysis of biomass for computational biology applications. Details of the original model construction were included in the previous report [39]; briefly, the model contained 213 reactions and 215 metabolites and was constructed to represent glucose and/or xylose metabolism for synthesis of major essential biomolecules (DNA, RNA, protein, lipid, carbohydrate). The current work extended the model to include the pathway for phenol degradation with the aid of MetaCyc, KEGG, and NCBI databases [40-42]. Genes for this pathway from the A. acidocaldarius genome were previously identified in [9]. Eight new reactions were added to the model to represent this pathway for a total of 221 reactions and 223 metabolites. This model allows for in silico probing of metabolism on different combinations of glucose, xylose, and phenol as carbon substrates, with possible byproducts of acetate, ethanol, formate, and lactate considered as common organic byproducts. CellNetAnalyzer $[43,44]$ was used to verify topological properties of the network (e.g., ensure appropriate connectivity and no blocked reactions) and to facilitate interconversion of model formats among SBML, COBRA Toolbox, and efmtool file formats. The Supplementary Materials include the Excel file with all model details and justifications including new reactions with enzyme documentation, as well as the SBML and COBRA Toolbox model files (Files S1-S3).

\subsection{Elementary Flux Mode Analysis and Ecological Analysis of EFMs}

Elementary flux modes (EFMs) were enumerated using efmtool [45] running within a MATLAB interface (https://csb.ethz.ch/tools/software/efmtool/documentation.html). Resource allocation analysis (i.e., cost efficiency assessment) of the resulting EFMs was performed with MATLAB processing by calculating the query costs (ratios of reactions, e.g., flux through carbon uptake reaction divided by flux through biomass reaction for each EFM in the simulation). Costs considered included carbon cost, oxygen cost, and pathway or enzyme investment as approximated by the sum of total (absolute) reaction fluxes. From the simulations for each carbon substrate combination (glucose, xylose, phenol, glucose + xylose, and phenol + xylose), two subsets of EFMs were analyzed-biomass-synthesizing pathways 
and energy-generating pathways. Costs were normalized across EFMs by scaling to the biomass flux (carbon moles of biomass synthesized) or energy flux (moles of excess ATP produced). Costs were then plotted against each other to examine a two-dimensional tradeoff space, and the convex hull function in MATLAB (convhull) was used to identify the perimeter of the tradeoff space and analyze the minimization of the tradeoff between the two costs (i.e., simultaneously minimizing costs along the tradeoff curve to determine the set of most efficient EFMs relating to the given costs). The EFMs on the tradeoff curve were extracted and exported to Excel for further analysis of the flux distributions in the most efficient pathways (e.g., byproduct profiles and pathway utilization). The MATLAB code used to process the EFM simulations is included in the Supplementary Materials (Files S4-S5).

\subsection{Dynamic Flux Balance Analysis}

A single flux balance analysis (FBA) simulation can be performed quickly for a genome-scale model with the MATLAB COBRA Toolbox. A temporally dynamic extension of FBA (dynamic FBA or dFBA) has previously been developed $[46,47]$ to integrate substrate uptake and product secretion kinetics with stoichiometric metabolic models to predict metabolism on a dynamic time scale, more suitable for comparison with batch growth data. DFBAlab software (http://yoric.mit.edu/dfbalab) $[47,48]$ was used to formulate a dynamic environment for flux balance simulations with xylose and phenol serving as possible carbon substrates to simulate the experimental setup performed in Aston et al. [9]. Kinetic expression parameters $\left(\mathrm{v}_{\max }\right.$ and $\mathrm{k}_{\mathrm{m}}$ ) for phenol were adapted for the simulation from measurements in Aston et al. [9] with units converted to match DFBAlab conventions, and kinetic parameters implemented for xylose remained the same as those used for Escherichia coli in the provided DFBAlab example script [47,49], as no specific parameters were available for A. acidocaldarius. Initial conditions were chosen to represent the experimental environment in [9], with $30 \mathrm{mM}$ xylose, $5 \mathrm{mM}$ phenol, and $0.01 \mathrm{~g} / \mathrm{L}$ biomass. Iterative optimizations were run with Gurobi as the solver. DFBAlab applies objective function optimization as typically performed in traditional FBA simulations [31], but it provides additional flexibility in tailoring simulations by allowing multiple objective functions to be specified and applied in sequential order during the optimization process. Carbon substrate uptake and biomass were applied as objective functions, and the order of application was tested to determine the effect on the optimization and simulation output. The MATLAB input scripts required for the DFBAlab algorithm to run (main.m, RHS.m, and DRHS.m) are included in the Supplementary Materials (Files S6-S8).

\section{Results}

\subsection{Simultaneous Utilization of Hexose and Pentose Sugars}

Most microbes typically consume carbon sources in sequential order of greatest preference in a phenomenon termed carbon catabolite repression or regulation, well-characterized in E. coli, which consumes glucose first (classic carbon catabolite repression [16]) or Pseudomonas aeruginosa, which consumes organic acids prior to sugars (reverse carbon catabolite repression [17]). However, A. acidocaldarius has previously been observed to consume two sugars simultaneously in continuous culture (glucose and xylose, arabinose and xylose, and fructose and glucose [8]). The presented computational analysis focuses on metabolic pathways for simultaneous glucose and xylose consumption (six-carbon and five-carbon sugars, respectively) as a unique metabolic feature that few microorganisms are reported to perform naturally [50,51]. Enumeration of elementary flux modes for the $A$. acidocaldarius metabolic network considering glucose and/or xylose as possible carbon substrates resulted in 1,106,863 total EFMs-345,024 EFMs with only glucose consumed; 209,408 EFMs with only xylose consumed; and 552,431 EFMs with both glucose and xylose consumed. These subsets were further divided into biomass-producing and ATP-generating subsets to examine the contributions of growth and energy metabolism and the effect under different carbon substrate regimes. 
Resource allocation analysis was used to examine how consumption of glucose and xylose affect biomass and energy production efficiency and pathway utilization, specifically with respect to carbon and oxygen (two key growth- and energy-limiting variables), as well as pathway investment (approximated by total pathway flux). Figure 1a shows oxygen consumption as a function of carbon substrate cost normalized to biomass production for three subsets of EFMs (glucose-only, xylose-only, and glucose- and xylose-consuming EFMs). Glucose was predicted to be a more efficient carbon source than xylose; this difference between hexose and pentose sugars is well substantiated due to the higher number of carbons and ATP generated via glycolysis. When glucose and xylose were co-utilized, the predicted efficiency matched that of glucose as a sole carbon substrate as shown by the superimposed blue and purple envelopes in the lower portion of Figure 1a. However, there were some differences in predicted pathway utilization. Pathways along the tradeoff curve (or minimization envelope) for simultaneous glucose and xylose consumption did not utilize the full pentose phosphate pathway, and the transketolase and transaldolase reactions (E.C. numbers 2.2.1.1 and 2.2.1.2) were not active due to xylulose-5-phosphate funneled into the pathway from xylose assimilation, preventing the need for these two additional enzymes and reactions.

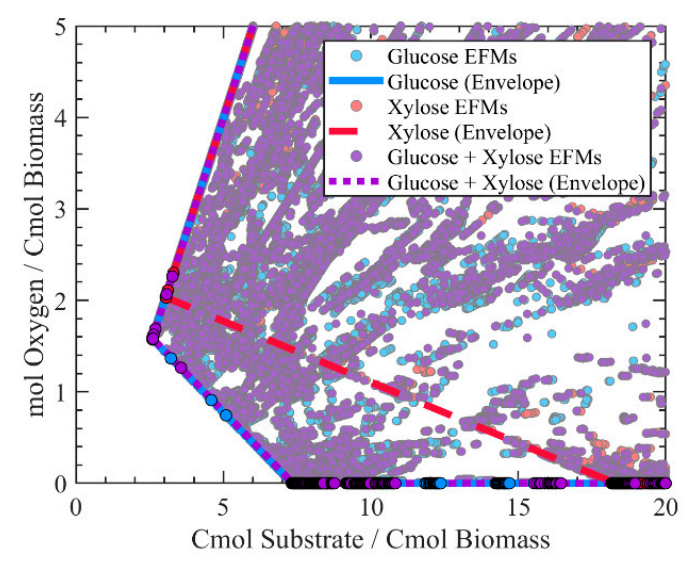

(a)

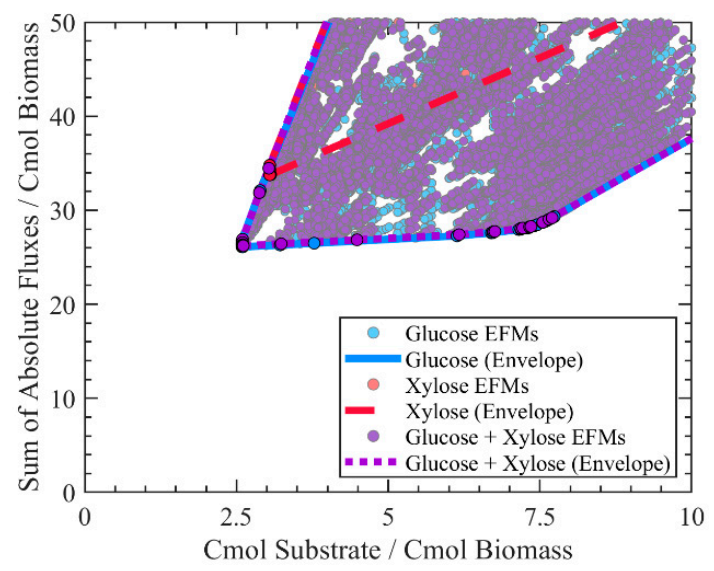

(b)

Figure 1. Glucose and xylose co-metabolism (glucose in blue; xylose in red; co-utilization in purple)-(a) oxygen cost as a function of carbon substrate cost (moles of oxygen versus moles of carbon consumed in substrate, normalized to moles of carbon in biomass); (b) pathway investment efficiency as a function of carbon substrate cost (sum of absolute fluxes per pathway versus moles of carbon consumed in substrate, normalized to moles of carbon in biomass). Each point on the plot represents the reaction ratio for an elementary flux mode (EFM) in the simulation; EFMs closer to the origin represent more efficient pathways.

When considering energy-generating EFMs, a slightly different phenomenon was observed in terms of the relationship among the three carbon substrate combinations-glucose was still predicted to be most efficient, but xylose as sole carbon substrate was predicted to be slightly more efficient than glucose and xylose co-metabolism (Figure 2a). Analyzing oxygen and substrate efficiency showed some additional differences to those observed above with biomass-the Entner-Doudoroff pathway was not predicted for either glucose or xylose as sole carbon substrates, but was predicted for glucose and xylose co-metabolism. Additionally, glucose or xylose as sole carbon substrates were both predicted to require a complete pentose phosphate pathway, but glucose and xylose co-metabolism did not predict use of the epimerase reaction interconverting xylulose-5-phosphate and ribulose-5-phosphate (E.C. number 5.1.3.1), again due to xylose assimilation. Additionally, use of the glyoxylate shunt was not predicted along the tradeoff curve for glucose and xylose co-metabolism, while it was predicted for glucose and xylose as sole carbon substrates. 


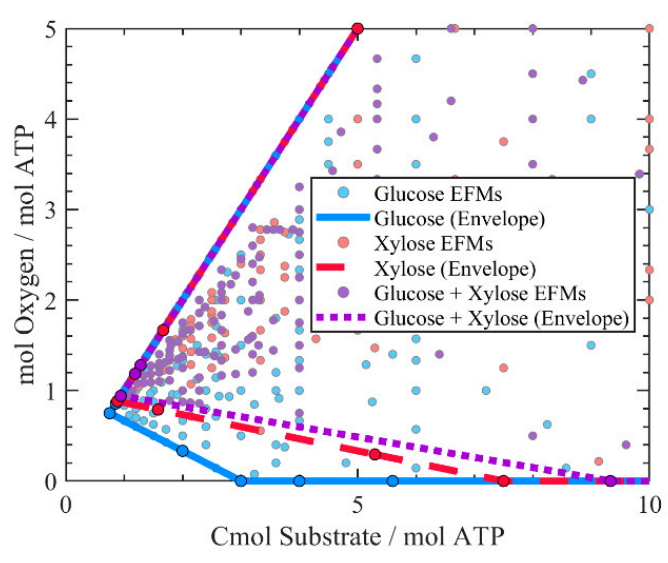

(a)

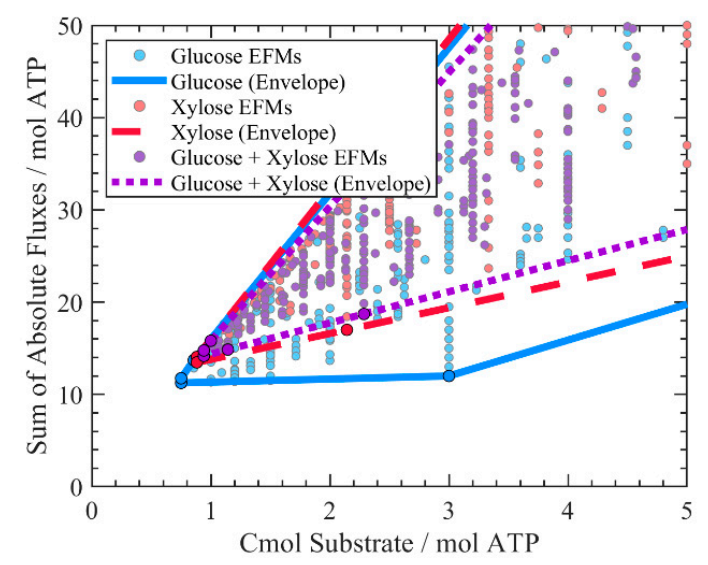

(b)

Figure 2. Glucose and xylose co-metabolism (glucose in blue; xylose in red; co-utilization in purple)-(a) oxygen cost as a function of carbon substrate cost (moles of oxygen versus moles of carbon consumed in substrate, normalized to moles of ATP generated); (b) pathway investment efficiency as a function of carbon substrate cost (sum of absolute fluxes per pathway versus moles of carbon consumed in substrate, normalized to moles of ATP generated). Each point on the plot represents the reaction ratio for an EFM in the simulation; EFMs closer to the origin represent more efficient pathways.

In addition to cellular operating costs such as carbon and oxygen, pathway or enzyme investment also plays an important role in dictating metabolic pathways [35,36]; the sum of total fluxes (absolute values) of the simulated EFMs was used as a proxy for pathway investment. Figure $1 \mathrm{~b}$ shows pathway investment as a function of carbon substrate. For both biomass-producing and energy-generating EFMs, a similar pattern was observed among carbon substrate combinations as seen for oxygen and substrate cost above-for biomass-producing pathways, glucose as a sole carbon substrate and co-consumption of glucose and xylose were equivalent and were more efficient than xylose as a sole carbon substrate, whereas for energy-generating EFMs, xylose as a sole substrate was predicted to be slightly more efficient than co-consumption of glucose and xylose, with glucose as a sole substrate predicted as the most efficient (Figure $2 b$ ). For pathway investment analysis, analogous patterns in usage of the pentose phosphate pathway were predicted as seen above, as well as the same pattern observed in the Entner-Doudoroff pathway utilization for energy-generating EFMs. However, the glyoxylate shunt was predicted to be used in all carbon substrate scenarios, and no byproducts were predicted for the energy-generating EFMs whereas acetate was the only organic byproduct predicted for the biomass-producing EFMs.

\subsection{Impact of Phenol Degradation}

Comparing phenol with glucose or xylose as singular carbon sources, phenol was predicted to be much less efficient than glucose or xylose, requiring both more carbon and substantially more oxygen as well as greater pathway investment, due to the key oxidation step required to utilize this compound (Figure 3). Availability of phenol as a sole substrate resulted in less variety in predicted organic byproducts (only acetate rather than including also ethanol, formate, and/or lactate), and use of the glyoxylate shunt was predicted to be necessary for efficient phenol consumption. 


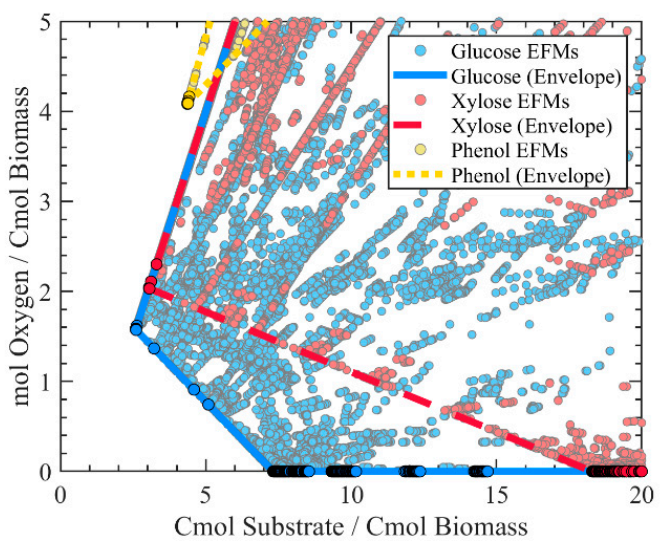

(a)

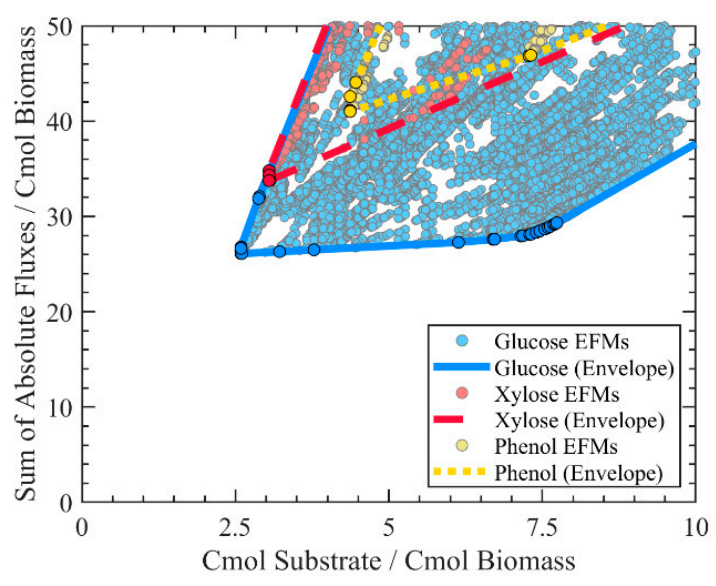

(b)

Figure 3. Glucose (in blue), xylose (in red), and phenol (in yellow) as singular carbon substrates-(a) oxygen cost as a function of carbon substrate cost (moles of oxygen versus moles of carbon consumed in substrate, normalized to moles of carbon in biomass); (b) pathway investment efficiency as a function of carbon substrate cost (sum of absolute fluxes per pathway versus moles of carbon consumed in substrate, normalized to moles of carbon in biomass). Each point on the plot represents the reaction ratio for an EFM in the simulation; EFMs closer to the origin represent more efficient pathways.

Considering the availability of both xylose and phenol as simultaneous substrates, the presence of xylose shifted the tradeoff curve much closer to that of xylose as a sole carbon substrate by offsetting the oxygen requirement with a comparatively much more energy efficient carbon source (Figure 4). Co-consumption of phenol and xylose resulted in a greater variety of predicted byproducts (all of acetate, ethanol, formate, and lactate, compared to only acetate for phenol as sole substrate or acetate, formate, and lactate for xylose as sole substrate). There was also more flexibility in the operation of the full TCA cycle with some reactions not required for efficient metabolism, e.g., fumarase reaction converting fumarate to malate (E.C. number 4.2.1.2) or malate dehydrogenase reaction converting malate to oxaloacetate (E.C. number 1.1.1.37). In terms of pathway investment, the glyoxylate shunt was also not predicted to be required under co-metabolism of phenol and xylose. 


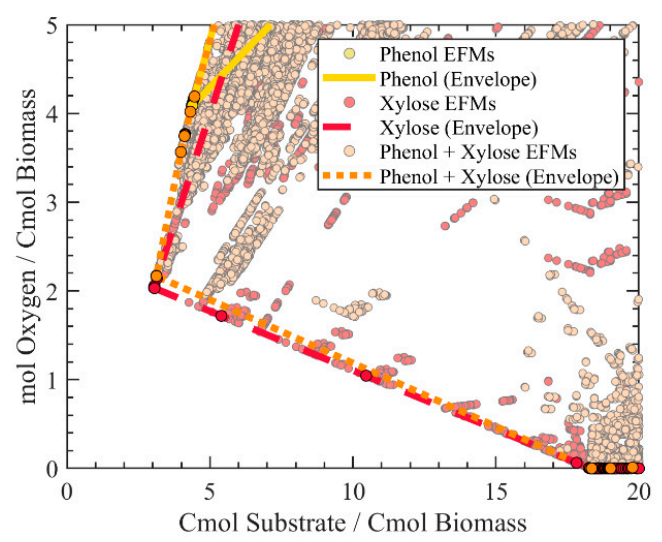

(a)

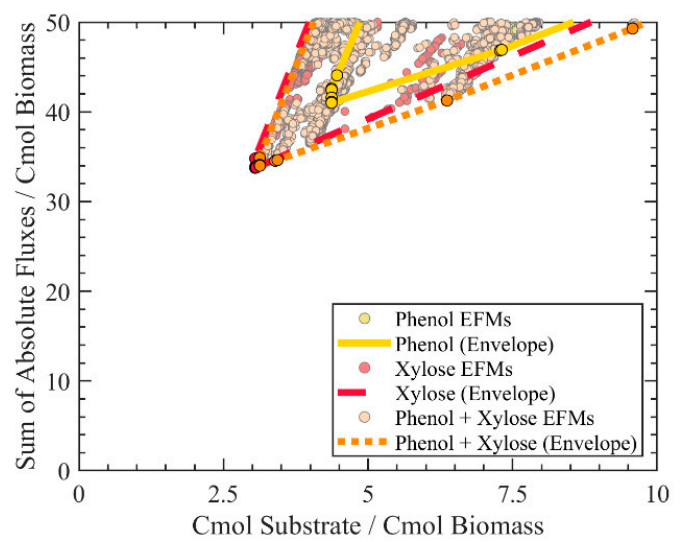

(b)

Figure 4. Xylose and phenol co-metabolism (phenol in yellow; xylose in red; co-utilization in orange)-(a) oxygen cost as a function of carbon substrate cost (moles of oxygen versus moles of carbon consumed in substrate, normalized to moles of carbon in biomass); (b) pathway investment efficiency as a function of carbon substrate cost (sum of absolute fluxes per pathway versus moles of carbon consumed in substrate, normalized to moles of carbon in biomass). Each point on the plot represents the reaction ratio for an EFM in the simulation; EFMs closer to the origin represent more efficient pathways.

\subsection{Dynamic Co-metabolism of Phenol and Xylose}

Upon examining the efficiencies of different carbon substrates and investigating the interaction effects of xylose and phenol as co-substrates, dynamic flux balance analysis (dFBA) was used to investigate the temporal aspect of co-metabolism. Previous research has demonstrated experimentally the ability of $A$. acidocaldarius to degrade phenol with xylose as the primary carbon substrate [9]. DFBAlab software $[47,48]$ was used to integrate kinetic expressions of xylose and phenol consumption with the A. acidocaldarius metabolic model to examine the effect of co-metabolism on biomass production and organic byproduct secretion over time. This simulation environment is more representative of batch growth. The kinetics of xylose and phenol consumption were described with classic Henri-Michaelis-Menten kinetics [52]. Rate and half-saturation constants ( $v_{\max }$ and $\mathrm{k}_{\mathrm{m}}$ ) were obtained from Aston et al. [9] for phenol using the experimental measurements performed with A. acidocaldarius and from Hanly and Henson [49] for xylose using measurements established for E. coli as an approximation. Typical FBA simulations employ a single objective function as the optimization criterion for the linear programming algorithm; however, DFBAlab software implementation of dynamic FBA uses lexicographic optimization to provide flexibility in the optimization criterion, allowing for multiple different objective functions to be specified and to be optimized in a hierarchical fashion [47]. Different permutations of multiple possible objective functions were tested, with the result that optimizing carbon substrate uptake first (xylose and phenol consumption) and secondarily optimizing for biomass production (growth) yielded simulation output that was more consistent with the batch growth patterns observed in Aston et al. [9] (Figure 5a). This resulted in predicted simultaneous consumption of xylose and phenol. The order of optimization for xylose and phenol uptake did not affect the simulation output (xylose optimized first or phenol optimized first). Byproduct secretion was also included in the optimization criteria as a tertiary objective (simultaneously minimizing production of acetate, ethanol, formate, and lactate), and the byproduct profiles predicted with co-metabolism followed a similar profile as predicted with elementary flux mode analysis, predicting predominantly lactate with smaller amounts of acetate and some ethanol and formate (Figure 5b). 


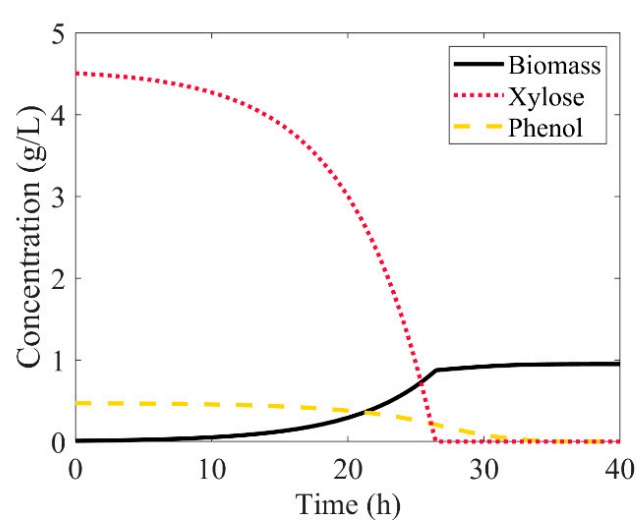

(a)

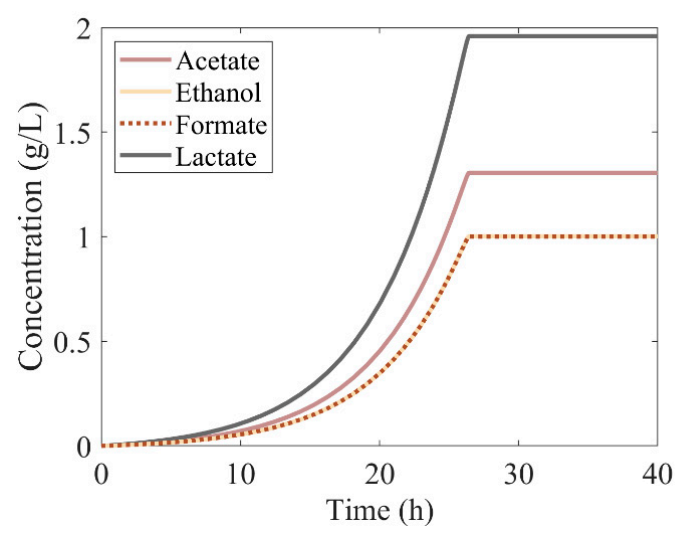

(b)

Figure 5. Dynamic flux balance analysis simulations of xylose and phenol co-metabolism in A. acidocaldarius with DFBAlab-(a) biomass (black), xylose (red), and phenol (yellow) as a function of time; (b) byproduct profiles resulting from the same simulation (lactate, acetate, and ethanol/formate, in order of decreasing concentration).

\section{Discussion}

The presented work examines in silico the metabolic network of $A$. acidocaldarius using two different computational perspectives to investigate the impact of substrate availability, particularly the influence of dual substrates, on predicted pathway utilization. Elementary flux mode analysis provides an unbiased view of the entire phenotype space; the resource cost analysis presented here narrows the phenotype space to investigate the cost efficiency of two typical operating costs, carbon uptake and oxygen utilization, as well as overall pathway investment into the predicted pathways. Minimizing costs and minimizing investment produces an ecological assessment predicting how the cellular metabolic network would operate to be competitive in its given environment $[37,53]$. This type of analysis is less amenable to extremely large genome-scale metabolic networks or community models, but can be performed as presented here using a smaller, more condensed network in which all EFMs can be enumerated.

Most microbes typically consume a single carbon source at a time due to regulation imposed by carbon catabolite repression [16]. However, A. acidocaldarius has been experimentally observed to consume multiple sugars simultaneously, including glucose and xylose [8], presenting an ideal opportunity to investigate co-metabolism of hexose and pentose sugars from a pathway perspective. Results showed, unsurprisingly, that glucose is a more efficient carbon substrate than xylose, and availability of both xylose and glucose did not predict greater efficiency than glucose as a sole substrate. However, the carbon and oxygen efficiencies under glucose as sole substrate compared with xylose and glucose as dual substrates were equivalent, indicating that co-metabolism does not reduce overall efficiency; minor pathway differences, such as differences in byproduct profiles as well as reducing or eliminating the need for some reactions in the pentose phosphate pathway, may also lend advantage to co-metabolism of substrates. Further research into details of genetic regulation and substrate kinetics as impacted by co-metabolism will add to the story.

The reported degradation of phenol and other phenolic compounds by A. acidocaldarius [9] also presents an ideal opportunity to investigate the interaction between phenol and sugar substrates from a pathway perspective. Phenol as a carbon source for growth is decidedly not an efficient substrate alone, even neglecting its toxic nature and considering only the metabolic network; a high quantity of oxygen is required to enter the degradative pathway (oxidase reaction). The availability of phenol with xylose as a co-substrate resulted in slightly lower predicted efficiency than xylose as a sole substrate, a different effect than that observed with glucose and xylose. However, metabolic efficiency 
is not greatly hindered by the addition of phenol, similar to the experimental observations seen in Aston et al. [9], where biomass accumulation was not affected until reaching higher levels of phenol.

The demonstrated case study for xylose and phenol co-metabolism presents an opportunity to merge constraint-based optimization methods with the presented EFM analyses to investigate the dynamic effects of co-metabolism and complement experimental data with time-course simulations. The DFBAlab simulations presented here illustrate the utility of the dynamic FBA framework for representing the batch growth scenario and describing substrate uptake, growth, and byproduct secretion over time. The increased variety in the byproduct profile was consistent with that predicted from EFM simulation analyses. The predicted time scale for simulated growth and consumption of substrate was slightly longer than observed in the experiments (experimental measurements were obtained over $25 \mathrm{~h}$, which was the entrance to the stationary phase [9], whereas the simulations showed biomass leveling out at $\sim 27 \mathrm{~h}$ ); this difference may be due to slight variations in biomass composition and/or maintenance energy parameters used in the model compared to the strain used in the experiment. Additional areas for continued improvement of the dynamic simulation framework include fine-tuning the xylose kinetic expression with strain-specific data, incorporating inhibition into the kinetic equations to better describe inhibition of phenol on overall metabolism (e.g., [54]), and expanding the model to include the influence of copper on phenol uptake (through multicopper oxidase enzymes [9]).

The presented work explores computationally the metabolism of a unique thermoacidophile and investigates the potential for expanding its role in lignocellulosic biomass processing beyond enzymatic pretreatment by examining sugar co-metabolism and inhibitory compound degradation. These capabilities could be employed for fermentative production of organic acids or in the development of microbial consortia for more effective and complete biomass processing. This study also demonstrates the promise for combining EFMA with dynamic FBA methods to gain deeper insight into microbial metabolism. In particular, this computational study highlights the versatility of the metabolism of A. acidocaldarius and its unique potential to co-metabolize substrates and degrade toxic compounds, elucidating underlying metabolic strategies and efficiencies, as well as the influence of environmental conditions. Substrate availability is a common limiting factor, with oxygen serving as a key limitation for obligate aerobes. A. acidocaldarius has high sensitivity to oxygen concentration; therefore, byproduct profiles could serve as an indicator of oxygen concentration and metabolic status in industry applications. The example of this study complements elementary flux mode analysis with flux balance analysis methods and may also serve as an encouragement to other researchers to expand the scope of computational analyses and bridge the gap between constraint-based modeling and metabolic pathway analysis methods, as each gains useful information and provides a unique approach.

Supplementary Materials: The following are available online at http://www.mdpi.com/2227-9717/8/5/502/s1, File S1: S1_Model_Justification.xlsx, File S2: S2_Model_SBML, File S3: S3_Model_CobraTb.mat, File S4: S4_EFM_Processing.m, File S5: S5_TSA.m, File S6: S6_main.m, File S7: S7_RHS.m, File S8: S8_DRHS.m.

Funding: This research was supported by the USDA National Institute of Food and Agriculture, Hatch 1018813.

Acknowledgments: The author would like to acknowledge administrative and mentoring support from the NC State Biological and Agricultural Engineering Department and mentors.

Conflicts of Interest: The author declares no conflict of interest.

\section{References}

1. Darland, G.; Brock, T. Bacillus acidocaldarius sp. nov., an acidophilic thermophilic spore-forming bacterium. Microbiology 1971, 67, 9-15.

2. Agafonov, D.E.; Rabe, K.S.; Grote, M.; Huang, Y.; Sprinzl, M. The esterase from Alicyclobacillus acidocaldarius as a reporter enzyme and affinity tag for protein biosynthesis. FEBS Lett. 2005, 579, 2082-2086. [CrossRef] [PubMed] 
3. Chandra, M.S.; Mallaiah, K.; Sreenivasulu, P.; Choi, Y.-L. Purification and characterization of highly thermostable $\alpha$-amylase from thermophilic Alicyclobacillus acidocaldarius. Biotechnol. Bioprocess Eng. 2010, 15, 435-440.

4. Guffanti, A.A.; Hou, E. The effect of $\mathrm{pH}$ on the passive proton conductance of Bacillus acidocaldarius. FEMS Microbiol. Lett. 1987, 41, 275-278. [CrossRef]

5. Yamazaki, Y.; Koyama, N.; Noso, Y. On the acidostability of an acidophilic thermophilic bacterium. Biochim. Biophys. Acta 1973, 314, 257. [CrossRef]

6. Mavromatis, K.; Sikorski, J.; Lapidus, A.; Del Rio, T.G.; Copeland, A.; Tice, H.; Cheng, J.-F.; Lucas, S.; Chen, F.; Nolan, M. Complete genome sequence of Alicyclobacillus acidocaldarius type strain (104-IA T). Stand. Genom. Sci. 2010, 2, 9. [CrossRef]

7. Wisotzkey, J.D.; Jurtshuk JR, P.; Fox, G.E.; Deinhard, G.; Poralla, K. Comparative sequence analyses on the $16 \mathrm{~S}$ rRNA (rDNA) of Bacillus acidocaldarius, Bacillus acidoterrestris, and Bacillus cycloheptanicus and proposal for creation of a new genus, Alicyclobacillus gen. nov. Int. J. Syst. Evol. Microbiol. 1992, 42, 263-269. [CrossRef]

8. Lee, B.D.; Apel, W.A.; DeVeaux, L.C.; Sheridan, P.P. Concurrent metabolism of pentose and hexose sugars by the polyextremophile Alicyclobacillus acidocaldarius. J. Ind. Microbiol. Biotechnol. 2017, 44, 1443-1458. [CrossRef]

9. Aston, J.E.; Apel, W.A.; Lee, B.D.; Thompson, D.N.; Lacey, J.A.; Newby, D.T.; Reed, D.W.; Thompson, V.S. Degradation of phenolic compounds by the lignocellulose deconstructing thermoacidophilic bacterium Alicyclobacillus acidocaldarius. J. Ind. Microbiol. Biotechnol. 2016, 43, 13-23. [CrossRef]

10. Sun, R.-C. Detoxification and separation of lignocellulosic biomass prior to fermentation for bioethanol production by removal of lignin and hemicelluloses. BioResources 2009, 4, 452-455.

11. Goto, K.; Tanaka, T.; Yamamoto, R.; Suzuki, T.; Tokuda, H. Characteristics of Alicyclobacillus. In Alicyclobacillus; Springer: Tokyo, Japan, 2007; pp. 9-48.

12. Eckert, K.; Schneider, E. A thermoacidophilic endoglucanase (CelB) from Alicyclobacillus acidocaldarius displays high sequence similarity to arabinofuranosidases belonging to family 51 of glycoside hydrolases. Eur. J. Biochem. 2003, 270, 3593-3602. [CrossRef] [PubMed]

13. Lee, H.-M.; Vo, P.; Na, D. Advancement of metabolic engineering assisted by synthetic biology. Catalysts 2018, 8, 619. [CrossRef]

14. Farrand, S.G.; Jones, C.W.; Linton, J.D.; Stephenson, R.J. The effect of temperature and pH on the growth efficiency of the thermoacidophilic bacterium Bacillus acidocaldarius in continuous culture. Arch. Microbiol. 1983, 135, 276-283. [CrossRef]

15. Farrand, S.G.; Linton, J.D.; Stephenson, R.J.; McCarthy, W.V. The use of response surface analysis to study the growth of Bacillus acidocaldarius throughout the growth range of temperature and pH. Arch. Microbiol. 1983, 135, 272-275. [CrossRef]

16. Görke, B.; Stülke, J. Carbon catabolite repression in bacteria: Many ways to make the most out of nutrients. Nat. Rev. Microbiol. 2008, 6, 613. [CrossRef]

17. Park, H.; McGill, S.L.; Arnold, A.D.; Carlson, R.P. Pseudomonad reverse carbon catabolite repression, interspecies metabolite exchange, and consortial division of labor. Cell. Mol. Life Sci. 2020, 77, 395-413. [CrossRef]

18. Behera, S.; Arora, R.; Nandhagopal, N.; Kumar, S. Importance of chemical pretreatment for bioconversion of lignocellulosic biomass. Renew. Sustain. Energy Rev. 2014, 36, 91-106. [CrossRef]

19. Mood, S.H.; Golfeshan, A.H.; Tabatabaei, M.; Jouzani, G.S.; Najafi, G.H.; Gholami, M.; Ardjmand, M. Lignocellulosic biomass to bioethanol, a comprehensive review with a focus on pretreatment. Renew. Sustain. Energy Rev. 2013, 27, 77-93. [CrossRef]

20. Kim, Y.; Ximenes, E.; Mosier, N.S.; Ladisch, M.R. Soluble inhibitors/deactivators of cellulase enzymes from lignocellulosic biomass. Enzym. Microb. Technol. 2011, 48, 408-415. [CrossRef]

21. Grabber, J.H. How do lignin composition, structure, and cross-linking affect degradability? A review of cell wall model studies. Crop Sci. 2005, 45, 820-831. [CrossRef]

22. Collins, L.D.; Daugulis, A.J. Biodegradation of phenol at high initial concentrations in two-phase partitioning batch and fed-batch bioreactors. Biotechnol. Bioeng. 1997, 55, 155-162. [CrossRef]

23. Gianfreda, L.; Iamarino, G.; Scelza, R.; Rao, M.A. Oxidative catalysts for the transformation of phenolic pollutants: A brief review. Biocatal. Biotransformation 2006, 24, 177-187. [CrossRef]

24. Michałowicz, J.; Duda, W. Phenols—Sources and toxicity. Pol. J. Environ. Stud. 2007, 16, 347-362. 
25. Kim, B.; Kim, W.J.; Kim, D.I.; Lee, S.Y. Applications of genome-scale metabolic network model in metabolic engineering. J. Ind. Microbiol. Biotechnol. 2015, 42, 339-348. [CrossRef]

26. Chen, P.-W.; Theisen, M.K.; Liao, J.C. Metabolic systems modeling for cell factories improvement. Curr. Opin. Biotechnol. 2017, 46, 114-119. [CrossRef]

27. Skraly, F.A.; Ambavaram, M.M.; Peoples, O.; Snell, K.D. Metabolic engineering to increase crop yield: From concept to execution. Plant Sci. 2018, 273, 23-32. [CrossRef]

28. Gu, C.; Kim, G.B.; Kim, W.J.; Kim, H.U.; Lee, S.Y. Current status and applications of genome-scale metabolic models. Genome Biol. 2019, 20, 121. [CrossRef]

29. Maarleveld, T.R.; Khandelwal, R.A.; Olivier, B.G.; Teusink, B.; Bruggeman, F.J. Basic concepts and principles of stoichiometric modeling of metabolic networks. Biotechnol. J. 2013, 8, 997-1008. [CrossRef]

30. Varma, A.; Palsson, B.O. Stoichiometric flux balance models quantitatively predict growth and metabolic by-product secretion in wild-type Escherichia coli W3110. Appl. Environ. Microbiol. 1994, 60, 3724-3731. [CrossRef]

31. Orth, J.D.; Thiele, I.; Palsson, B.Ø. What is flux balance analysis? Nat. Biotechnol. 2010, 28, 245. [CrossRef]

32. Trinh, C.T.; Wlaschin, A.; Srienc, F. Elementary mode analysis: A useful metabolic pathway analysis tool for characterizing cellular metabolism. Appl. Microbiol. Biotechnol. 2009, 81, 813. [CrossRef] [PubMed]

33. Zanghellini, J.; Ruckerbauer, D.E.; Hanscho, M.; Jungreuthmayer, C. Elementary flux modes in a nutshell: Properties, calculation and applications. Biotechnol. J. 2013, 8, 1009-1016. [CrossRef] [PubMed]

34. Jungreuthmayer, C.; Gerstl, M.P.; Navarro, D.A.P.; Hanscho, M.; Ruckerbauer, D.E.; Zanghellini, J. Designing optimized production hosts by metabolic modeling. In Metabolic Network Reconstruction and Modeling; Humana Press: New York, NY, USA, 2018; pp. 371-387.

35. Carlson, R.P. Metabolic systems cost-benefit analysis for interpreting network structure and regulation. Bioinformatics 2007, 23, 1258-1264. [CrossRef] [PubMed]

36. Carlson, R.P. Decomposition of complex microbial behaviors into resource-based stress responses. Bioinformatics 2009, 25, 90-97. [CrossRef] [PubMed]

37. Beck, A.; Hunt, K.; Bernstein, H.C.; Carlson, R. Interpreting and designing microbial communities for bioprocess applications, from components to interactions to emergent properties. In Biotechnology for Biofuel Production and Optimization; Elsevier: Amsterdam, The Netherlands, 2016; pp. 407-432.

38. Gebbie, L.; Dam, T.T.; Ainscough, R.; Palfreyman, R.; Cao, L.; Harrison, M.; O'Hara, I.; Speight, R. A snapshot of microbial diversity and function in an undisturbed sugarcane bagasse pile. BMC Biotechnol. 2020, 20, 1-16. [CrossRef]

39. Beck, A.E.; Hunt, K.A.; Carlson, R.P. Measuring cellular biomass composition for computational biology applications. Processes 2018, 6, 38. [CrossRef]

40. Caspi, R.; Billington, R.; Ferrer, L.; Foerster, H.; Fulcher, C.A.; Keseler, I.M.; Kothari, A.; Krummenacker, M.; Latendresse, M.; Mueller, L.A. The MetaCyc database of metabolic pathways and enzymes and the BioCyc collection of pathway/genome databases. Nucleic Acids Res. 2016, 44, D471-D480. [CrossRef]

41. Kanehisa, M.; Goto, S.; Sato, Y.; Furumichi, M.; Tanabe, M. KEGG for integration and interpretation of large-scale molecular data sets. Nucleic Acids Res. 2012, 40, D109-D114. [CrossRef]

42. Coordinators, N.R. Database resources of the National Center for Biotechnology Information. Nucleic Acids Res. 2017, 45, D12.

43. Klamt, S.; Saez-Rodriguez, J.; Gilles, E.D. Structural and functional analysis of cellular networks with CellNetAnalyzer. Bmc Syst. Biol. 2007, 1, 2. [CrossRef]

44. von Kamp, A.; Thiele, S.; Hädicke, O.; Klamt, S. Use of CellNetAnalyzer in biotechnology and metabolic engineering. J. Biotechnol. 2017, 261, 221-228. [CrossRef] [PubMed]

45. Terzer, M.; Stelling, J. Large-scale computation of elementary flux modes with bit pattern trees. Bioinformatics 2008, 24, 2229-2235. [CrossRef] [PubMed]

46. Mahadevan, R.; Edwards, J.S.; Doyle III, F.J. Dynamic flux balance analysis of diauxic growth in Escherichia coli. Biophys. J. 2002, 83, 1331-1340. [CrossRef]

47. Gomez, J.A.; Höffner, K.; Barton, P.I. DFBAlab: A fast and reliable MATLAB code for dynamic flux balance analysis. BMC Bioinform. 2014, 15, 409. [CrossRef] [PubMed]

48. Gomez, J.A.; Barton, P.I. Dynamic flux balance analysis using DFBAlab. In Metabolic Network Reconstruction and Modeling; Humana Press: New York, NY, USA, 2018; pp. 353-370. 
49. Hanly, T.J.; Henson, M.A. Dynamic flux balance modeling of microbial co-cultures for efficient batch fermentation of glucose and xylose mixtures. Biotechnol. Bioeng. 2011, 108, 376-385. [CrossRef] [PubMed]

50. Kim, J.-H.; Block, D.E.; Mills, D.A. Simultaneous consumption of pentose and hexose sugars: An optimal microbial phenotype for efficient fermentation of lignocellulosic biomass. Appl. Microbiol. Biotechnol. 2010, 88, 1077-1085. [CrossRef]

51. Lin, L.; Song, H.; Tu, Q.; Qin, Y.; Zhou, A.; Liu, W.; He, Z.; Zhou, J.; Xu, J. The Thermoanaerobacter glycobiome reveals mechanisms of pentose and hexose co-utilization in bacteria. PLoS Genet. 2011, 7, e1002318. [CrossRef]

52. Cornish-Bowden, A.; Mazat, J.-P.; Nicolas, S. Victor Henri: 111 years of his equation. Biochimie 2014, 107, 161-166. [CrossRef]

53. Carlson, R.P.; Oshota, O.J.; Taffs, R.L. Systems analysis of microbial adaptations to simultaneous stresses. In Reprogramming Microbial Metabolic Pathways; Springer: Dordrecht, The Netherlands, 2012; pp. 139-157.

54. Han, K.; Levenspiel, O. Extended Monod kinetics for substrate, product, and cell inhibition. Biotechnol. Bioeng. 1988, 32, 430-447. [CrossRef]

(C) 2020 by the author. Licensee MDPI, Basel, Switzerland. This article is an open access article distributed under the terms and conditions of the Creative Commons Attribution (CC BY) license (http://creativecommons.org/licenses/by/4.0/). 